\title{
La apuesta por los ciudadanos
} Michelle y Armand MATTELART

Michelle y Armand Mattelart vivieron 11 años en Chile y fueron expulsados con el golpe del 11 de septiembre de 1973. Regresaron por primera vez en 1991 y en el año 2000, en su segunda vuelta, tuvieron un diálogo con académicos y estudiantes de nuestra Escuela de Periodismo en el local de calle Periodista José Carrasco Tapia. Armand Mattelart fue entrevistado después por Comunicación y Medios ${ }^{1}$ y este año, con motivo del tercer retorno de él y Michelle a Chile, tuvimos oportunidad de reanudar el diálogo.

El encuentro se produjo el viernes 8 de julio en el estudio de televisión de nuestro Instituto de la Comunicación e Imagen, ante un público de académicos, investigadores y estudiantes. Fue un diálogo amplio y de temario abierto, que se abrió con las renovadas emociones del regreso y las inevitables comparaciones entre el Chile de 1973 y el de hoy. Un ejercicio que no solo apostó a la memoria, sino que también permitió lecturas actuales sobre el género, la comunicación, la diversidad y la sociedad de la información.

\section{Desde "Onda" a "Machuca"}

Michelle Mattelart: Les voy a dar dos detalles sobre este nuevo viaje nuestro a Chile que me parecen muy sugestivos y que tienen una implicación personal. Vimos aquí en Santiago la película "Machuca", una muy buena película, la vimos con mucha emoción. Resulta que en la película, al principio, se recuerda en un quiosco la revista Onda, publicada por la Editorial Quimantú, en la cual me tocó trabajar. Y entonces para mí fue realmente la impresión de que se reempezaba. Había un retorno de la memoria, no había ya la memoria como recuerdo. Es decir jugaba, estaba funcionando, se estaba renovando.

El segundo detalle es siempre a raíz de Onda, una revista que se podía llamar alternativa dentro del mundo juvenil, contraponiéndose a las revistas de ídolos. Y este segundo detalle tiene una incidencia mucho más dolorosa, pero remite también a las cosas que pasaron. En la revista Rocinante de una de las últimas semanas leí un artículo de María Eugenia Camus, una periodista que yo conocí muy bien. Un articulo donde ella recordaba la risa, la sonrisa de Diana Arón, que fue una periodista que yo también conocí muchísimo, que trabajó conmigo y con varias otras y otros en la revista Onda. Y María Eugenia Camus recordó en Rocinante el trabajo de Diana Arón en la revista Onda. Diana fue torturada por la DINA y murió ${ }^{2}$. Todo esto me hace decir que la memoria se está recordando y para mí este es el sello de este Chile de hoy.

Armand Mattelart: Chile nunca ha dejado de formar parte de mí. Estuvimos con Michelle 11 años en Chile (entre 1963 y 1973) trabajando en diversas instituciones. Durante 17 años en que no pudimos volver a Chile, después de la expulsión en septiembre de 1973, tuve casi cada día una pesadilla sobre el golpe de Estado, sobre toda la represión, y bastó que volvira

\footnotetext{
${ }^{1}$ Armand Mattelart: El espejo universal. Por Carlos Ossa Swears. Comunicación y Medios No 13. Año 2002.

${ }^{2}$ La periodista Diana Arón, militante del Movimiento de Izquierda Revolucionaria (MIR), fue arrestada el 18 de noviembre de 1974 en Santiago y se le vio por última vez con vida en enero de 1975 en el campo de prisioneros de Cuatro Alamos. El juez Alejandro Solís pidió condenas de 15 años de presidio para el general retirado Manuel Contreras y el brigadier Miguel Krasnoff, y de 10 años para los coroneles Pedro Espinoza y Marcelo Morén y la misma pena para el agente civil Osvaldo Romo, como autores de la desaparición de la periodista, pero el 1 de junio de 2005 la quinta sala de la Corte de Apelaciones revocó la pena y les aplicó la ley de amnistía de 1978 en un fallo de dos votos contra uno.
} 
en 1991, cuando levantaron la interdicción, y pisar el suelo de este país, para que se esfumaran completamente esas pesadillas.

Viví entonces durante 17 años con estos recuerdos que estaban entremezclados de represión, pero también de pérdidas de amigos íntimos. Entre ellos una persona que trabajaba con nosotros, con el cual redactamos el primer libro sobre comunicación masiva y revolución socialista, que era un argentino, Patricio Viedma. Cuando volvimos en 1991 casi no pudimos encontrar a nadie. Finalmente recorrimos los lugares donde habíamos vivido, vimos algunas personas y fue una terapia, una verdadera terapia, y volvimos en 2000 por segunda vez.

Es muy interesante, porque esta segunda vez la vuelta a Chile fue proporcionada por otra generación que la nuestra. Era la generación de nuestros hijos. Quienes nos convidaron fue la gente de la Editorial LOM, Pablo Slachevsky, quien hizo sus estudios en el exilio con nuestros hijos en Francia. Entonces, para nosotros era comprobar como la diáspora, a pesar de todos los sufrimientos que representa el exilio, entraba a trabajar también la realidad chilena. Un periodista muy atrevido me preguntó "usted qué piensa de estos que se exiliaron, que nos dieron lecciones y después se fueron". Yo le dije: mira muchos de los que se fueron de Chile es porque los expulsaron o no pudieron volver.

Nos animó mucho en el año 2000, porque fue el primer reencuentro finalmente con un Chile donde se veían reaparecer luces de esta conciencia critica que fue para mí una característica de la cultura chilena. En la cultura chilena que vivimos nosotros durante los 11 años en que estuvimos aquí, siempre nos sorprendió esta viveza, esta intuición de lo político, este espíritu critico que encontramos también en Francia.

Hoy volvemos, estamos aquí. La sensación que tengo es que para mí este reencuentro es otra sensación. Reencuentro el pueblo chileno a través de caras: esta vez a lo que me dediqué es a mirar las caras. Porque finalmente esto es una continuidad también, es una continuidad que nos recuerda nuestra vivencia, nuestro compromiso con la realidad chilena. Porque de no haber sido expulsados estaríamos aquí para siempre. Habíamos decidido quedarnos para siempre en Chile y nuestros hijos eran "más porotos que los chilenos".

Para mí Chile fue básico y eso no es retórica. Fue importante, porque realmente a Michelle y a mí la realidad chilena nos construyó a nivel de nuestras intuiciones políticas, muy vinculadas a la manera de cómo concebimos nuestro trabajo de investigar. Creo que nuestra vivencia en Chile, nuestra vivencia de más de 10 años, nos permitió finalmente entender dos cosas que, por lo menos de mi lado, no hubiera podido intuir quedándome en Europa. Es, primero, la importancia que tienen los medios y los dispositivos ideológicos en la construcción finalmente de alternativas. Me recordaré siempre que llegado a Francia había un abismo entre lo que habíamos vivido a nivel incluso de la realidad concreta de los medios, con la oposición virulenta al gobierno de la Unidad Popular, pero también los déficit de respuesta de la izquierda. Había un desfase entre lo que habíamos vivido en Chile y el estado de reflexión que verdaderamente era muy débil de la izquierda francesa, y en general europea, en la cuestión de los medios.

La segunda cosa fue una intuición magistral que nos reveló la realidad chilena durante el gobierno de la Unidad Popular y eso lo vi muy tardíamente. Y es lo que ocurrió en Chile a nivel del forcejeo internacional en contra del proceso de reformas de la Unidad Popular a través de las campañas de agencias y otros medios de prensa. Finalmente, después de tantos años, yo me digo que si algunos hacen empezar la era de la globalización en los años 80, 90, la batalla y la entrada realmente en la esfera global se vio de manera 
muy embrionaria en todos los intentos de resistencia de los tres años de la Unidad Popular. A este contexto, Salvador Allende lo llamaba el cerco ideológico alrededor de Chile. Y para mí una de las piezas mayores de denuncia y de análisis crítico de lo que iba a ser la globalización después es su discurso en las Naciones Unidas en contra de las multinacionales de la época ${ }^{3}$.

\section{Género, revistas femeninas y comunicación}

Michelle Mattelart: Ha habido muchos cambios entre el momento en que vivimos y los años en que comencé a trabajar el tema de la mujer, de las revistas consagradas al universo de las mujeres. Pero en un primer momento quiero recalcar justamente que una investigadora irlandesa, que tiene una fama muy grande en el ámbito internacional, Margaret Gallagher, cuando hizo el prólogo de una nueva revista feminista que ha sido publicada en Estados Unidos y en Inglaterra, sobre feminismo y medios, ella subrayó dos trabajos como las piedras fundadoras de un acercamiento critico a los medios vinculados con el universo femenino.

Los trabajos que mencionó y subrayó Margaret Gallagher fueron el artículo que escribí, que fue realmente fundador creo yo y que sigue siendo valido, "Apuntes sobre lo moderno: una manera de leer la revista femenina" Adriana Santa Cruz, "Compropolitan", , pocos años después. Esto muestra que estos trabajos tuvieron alguna repercusión, surtieron algunos efectos.

Ahora ha cambiado muchísimo. Hubo la mediación de todo el movimiento de auge de la conciencia de las mujeres. Cuando yo viví en Chile realmente no se daba esta conciencia. Me acuerdo que yo venía muy joven de Francia, donde no había tenido el tiempo de acercarme mucho a lo que ya era un movimiento feminista. Son los años 70 sobre todo los años clave del desarrollo del movimiento feminista en varios países, pero en Francia ya había empezado paulatinamente en los años 60.

Pero aquí en Chile me acuerdo que no había ninguna conciencia de la dominación peculiar que sufre la mujer. Todavía no se hablada de género, eso apareció, se consolidó, en los años 90 sobre todo. Y había en los años 60 ciertas actitudes en los partidos políticos de izquierda chilena que llamaban la atención de una mujer.

Pero ahora me parece que la situación ha cambiado muchísimo y quizás es el tema que ha alcanzado un nivel de profundización más alto en el ámbito universitario, académico, pero también en el ámbito de la movilización en los países latinoamericanos y Chile está dentro de este ámbito. Creo que gracias a este tema de género los países latinoamericanos, Norteamérica y países europeos se están comunicando. Es decir, compartiendo bibliografías, avances, etcétera. Evidentemente que con criticas también, porque el movimiento avanza y propone lecturas complejas de lo que pasa en este dominio, muy importante desde el punto de vista de la penetración comercial. El mundo de las revistas femeninas y de los programas audiovisuales femeninos es uno de los que más surte beneficios, el más vinculado al auge de la mentalidad consumista. Aunque cada vez más

\footnotetext{
${ }^{3}$ Salvador Allende habló ante la Asamblea General de la Organización de Naciones Unidas en Nueva York el 4 de diciembre de 1972. El discurso está disponible en http://www.abacq.net/imagineria/cronolo4.htm Consultado el 19/09/2005.

${ }^{4}$ MATTELART, Michelle: “Apuntes sobre lo moderno: una manera de leer la revista femenina”. En: Casa de las Américas. Año XIII. No. 77. Marzo-abril. 1973. pp. 112-125.

${ }^{5}$ ERAZO, Viviana y SANTA CRUZ, Adriana. Compropolitan. El orden transnacional y su modelo femenino: Un estudio de las revistas femeninas en América Latina. Editorial Nueva Imagen. México, 1980.
} 
sectores, el sector del niño, el sector del hombre, están llamados a concurrir muy fuertemente a esta consolidación de esta mentalidad consumista.

Creo que hay unas lecturas criticas que avanzan mucho, pero lo que hay que decir es que parece que cada vez más se consolida también una visión alienada de la mujer. Los análisis críticos son muy interesante, lúcidos, pero están muy sofisticados. No hay comunicación entre estos análisis y lo que pasa en el ámbito globalizado de los medios, donde este sector del consumismo orientado a la mujer sigue siendo de un avance fuertísimo.

\section{Complejos comunicacionales y homogenización cultural}

Armand Mattelart: Siempre estoy un poco molesto cuando debo hablar de ese tema. Pienso fundamentalmente que es difícil hablar de este tema, de complejo mediático, económico, político, porque de eso hay que hablar, y también militar, como se ve en la guerra de Irak.

Es difícil de hablar de esta variable, que llamaríamos la dimensión estructural de los sistemas de comunicación, porque el gran problema (y eso es un elemento que favorece el no entendimiento de las apuestas en el campo de la comunicación por los ciudadanos) es que cuando se habla de complejos se habla de estructuras, el ciudadano y la ciudadana común finalmente tienen miedo. Lo estructural muchas veces está interpretado, cuando no se tiene la información, el conocimiento, como finalmente un análisis que demoniza este orden de la comunicación.

Toda mi vida, desde mi primer libro sobre "Agresión desde el espacio", he tratado de luchar en contra de esta aprensión, de este entendimiento reducido de lo que es la estructura. Creo que hoy en día tenemos un problema muy serio en hacer pasar estos análisis de tal manera que la gente, los ciudadanos, se sientan concernidos, implicados, que tienen algo que ver con ellos.

Entonces lo que está cambiando, y eso se puede decir desde los años 90, es que progresivamente estas apuestas estructurales se vuelven temas de movilización, de toma de conciencia, de movimientos sociales y de movimientos ligados a movilización de organizaciones profesionales de la cultura. Es importante porque ya está estableciéndose la mediación entre unos análisis superestructurales y finalmente la gente.

Es verdad que la cuestión de la Convención sobre la Diversidad Cultural que se discute hoy en la Unesco es una apuesta estructural fundamental, ya que la diversidad cultural es ir en contra de todas estas lógicas de concentración que hacen que los países estén desprovistos de casas editoriales nacionales o locales. Diversidad es ir contra todo lo que se refiere a la pérdida de identidad a través de estos procesos de concentración.

Es interesante ver que hoy en día se están tejiendo pasarelas entre temas que hace apenas cinco años, cuando vinimos aquí en el año 2000, estaban todavía abstractos. Tomo dos ejemplos, el de la Convención para la Diversidad Cultural, donde uno ve ahora una coalición chilena para la defensa de la diversidad cultural, que fue pionera en la construcción de una red mundial con los canadienses y que defienden la idea de políticas publicas en materia de libros, de televisión, de cine, etcétera. Eso es fundamental.

Y otro foco, donde la cultura no entra por la gran puerta como en la Unesco, pero entra por la ventana, es el de todos los temas referidos a otro logo, que se llama la Sociedad

\footnotetext{
${ }^{6}$ MATTELART, Armand. Agresión desde el espacio. Cultura y napalm en la era de los satélites. Editorial Siglo XXI, 1972.
} 
de la Información. Es muy interesante ver que los movimientos sociales metidos en la cuestión de la comunicación o las coaliciones profesionales se vuelven a encontrar ahora en cada frente. Pueden encontrarse tanto para oponerse a una concepción de la propiedad intelectual que signifique cada vez más poder a los monopolios del saber y a los medios, como discutir sobre la preservación, por ejemplo, del artesanado, de las artes y espectáculos en cada realidad.

Lo que me parece importante es decir que estos análisis estructurales durante mucho tiempo se quedaron como superestructurales, y ahora los movimientos empiezan a apropiarse de lo que parecía abstracto. La noción de sociedad de la información es totalmente abstracta para la gente. ¿Qué quiere decir? Lo que es interesante ver es que los movimientos sociales han logrado hacer bajar la noción en la cotidianidad. ¿Y qué es la cotidianidad? Significa que para que haya una verdadera sociedad de la información debe haber diversidad lingüística, diversidad cultural, que quiere decir diversidad mediática. Es legitimar el establecimiento en los sistemas de comunicación nacionales de un tercer sector, una necesidad de revisar la noción de servicio publico que está en crisis, necesidad de luchar en contra de la concentración en las industrias culturales. Entonces, está avanzando poco a poco.

No por ello hay que levantar las banderas de triunfo. Pienso que son temas extremadamente difíciles de hacer entrar en la conciencia social: es más fácil hacer salir la gente para ir en contra de la reforma de las pensiones, o las jubilaciones, que asumir la cuestión de los medios y de la cultura. Se interesan por el momento en la cultura, y se ve claramente en América Latina, los grupos que han invertido mucho en la reflexión critica sobre los medios o sobre la cultura y sectores de la población profesional. Creo que es importante decir esto, porque estimo que esta conciencia se va a expandir en círculos concéntricos progresivos.

Nada de triunfalismo. El proceso es lento, lo veo concretamente en la experiencia que tengo en Francia, donde creamos hace dos años un observatorio de los medios, tomando la idea avanzada en el Foro Social Mundial de Porto Alegre 2002. Pero se ve que es muy difícil, incluso cuando existe un movimiento social fuerte como ATTAC ${ }^{7}$. Es difícil hacer pasar la idea de que es necesario construir un contra poder perenne para que los ciudadanos se apoderen de este desafío de los medios.

El problema es allá porque no podemos satisfacernos con realizar campañas, movilizaciones, etcétera. Lo importante para este siglo es precisamente establecer un contra poder permanente, de tal manera que esta concentración, estos procesos de concentración, no desborden completamente la democracia.

El Parlamento Europeo, por ejemplo, se ha inquietado de la situación en Italia. Es fundamental discutir y poner al centro de un debate sobre los medios la cuestión de la concentración: qué es un monopolio, que es el umbral de la concentración, cuáles son las participaciones cruzadas, políticas, económicas. Si no pararemos en un verdadero atolladero.

Es fundamental, y no se puede discutir de diversidad cultural sin tomar este problema medular de la concentración. Les voy a dar un ejemplo que muestra cuán difícil es plantear este problema de la concentración. En los años 70, en la Unesco sacaron muchos informes, entre ellos el Informe McBride, que ustedes conocen, sobre el nuevo orden informativo mundial. Y en el centro del Informe McBride y en todo el debate en la

\footnotetext{
${ }^{7}$ ATTAC: Asociación para una Tasa a las Transacciones Financieras y la Acción Ciudadana.
} 
Unesco estaba el problema de la internacionalización, como se decía, y de la concentración. Yo sostengo que hoy en día es imposible plantear desde la Unesco el problema de la concentración. Le van a hablar mucho de las políticas culturales, pero saltan todo lo que se refiere a política de comunicación. ¿Así, cuando se vuelve con el tema de concentración? Entonces, quienes pueden plantear ese problema y quienes pueden desbordar finalmente la Unesco y otras organizaciones internacionales son los ciudadanos y es por eso que la organización de los ciudadanos en este campo es fundamental.

\section{Contra poder, una tarea de largo aliento}

Armand Mattelart: Primero, no hay que desesperarse. Todavía hay niveles de conciencia que están formándose. Es verdad que no hay posibilidad de establecer una presión para que la concentración de los medios se vuelva un tema del espacio publico y de la democracia sin el respaldo de ciudadanos conscientes. Doy el ejemplo de Italia. En Italia trataron de crear un ATTAC y no resultó. La cuestión del observatorio de medios, que está apoyado por Inter Press Service, esta agencia alternativa creada en los años 60, tiene dificultad de instalarse porque están divididos dentro de la propia izquierda o de las fuerzas progresistas.

Creo que eso demuestra que la cuestión de los medios es primero una cuestión de poder, de poder mediático, económico o político y que para enfrentarlo debe surgir también progresivamente una fuerza, un contrapoder. Pero eso realmente toma tiempo. Toma tiempo pero es significativo si miramos América Latina hoy, y yo podría hacer el mismo cuadro en Europa.

Tomo América Latina. Miremos las iniciativas que han surgido de la sociedad civil organizada, o descontenta si se quiere, frente a la concentración. Observemos México, donde no se puede hablar de la prensa y de la televisión como quinto poder o como primer poder, los ciudadanos han formulado propuestas que han sido rechazadas por el establishment, pero después de tantos años han formulado estas propuestas y acaban ahora de reconocer algunas radio comunitarias, entre ellas indígenas.

Miremos lo que ocurre en Argentina, donde todo este movimiento de radios comunitarias esta en la avanzadilla para reclamar un cambio en todo el sistema educativo.

Miremos lo que ocurre en Brasil. Es muy interesante en Brasil la contradicción del Ministerio de la Cultura, de Gilberto Gil, que quiere realmente avanzar en la Convención de la Diversidad Cultural y en la reformulación del sistema de comunicación en función de una extensión a la sociedad civil y a los grupos comunitarios, y el Ministerio de Comunicación, del mismo gobierno de Lula, que apoya a la cadena O Globo. Y cada vez que hay que definir en la nueva ley la noción de monopolio u oligopolio, interviene para que quede totalmente vaga, es decir inútil jurídicamente.

Yo creo que es una tarea de largo aliento y pienso que realmente la única manera de no ver la situación de manera apocalíptica es pensar que cada vez más gente va a ser concernida por esta cuestión. No es una cuestión solamente de especialistas, es una cuestión de vida cotidiana, sobre todo porque nos hablan tanto de sociedad del saber, de sociedad de la información, y no hay la más mínima condición para realizarla.

Cómo vamos a alcanzar sociedades del conocimiento si no planteamos todo el proceso de concentración que trabajan los medios. Es una estafa. Yo entiendo la impaciencia por lo que esta detrás de todo esto. El problema laboral de las nuevas generaciones yo lo sufro tanto, porque en Francia misma formamos y formo gente en doctorados, postgrados, y desembocan en un mercado de trabajo donde tienen un puesto 
precario. El problema es allá también y yo creo que va a ser fundamental en las luchas sindicales. No hay que olvidar nunca que los sindicatos de periodistas deberían ser los que incentiven estas luchas junto al movimiento social.

\section{Consumo y oferta mediática}

Michelle Mattelart: La crisis en la lectura de diarios, de revistas quizás sea muy intensa en Chile, pero no se puede generalizar. Tampoco es un hecho que los medios más segmentados se estén imponiendo. Tomemos la televisión abierta, como se la llama en Chile, expresión que me parece inapropiada, porque no es tan abierta. Pero si consideramos esta famosa televisión abierta, creo que marca hitos en el día, marca realmente una agenda del consumo, estructuras en la agenda de la cotidianidad fuera del trabajo.

Pienso que el medio masivo, al contrario de los que lo consideran en crisis, sigue teniendo una audiencia, sigue teniendo importancia y crea un universo simbólico. Creo justamente que el problema esta ahí como expresión de la globalización y sus referentes simbólicos. De todos modos habría que matizar, pero todo lo que se conoce sobre el impacto global de las telenovelas nos remite a formatos que están basados sobre el efecto de la emoción y que congregan a las familias. Esto sigue un dato muy importante.

Es cierto que gracias a las tecnologías hay una diversidad, una comunicación de punto a punto, una comunicación de organizaciones civiles, de establecimiento de redes. Pero el concepto de diversidad esta muy bien trabajado también por las cadenas masivas. Escuchamos en el simposio sobre diversidad cultural en la Universidad Católica ${ }^{8}$ respuestas como la del presidente del Directorio de Televisión Nacional (Carlos Mladinic), quien afirmó que el concepto de diversidad dentro de un medio o servicio publico está muy bien respetado en ese canal, porque tiene programas para cada sector de la población, para cada gusto.

Pero eso para mí no es diversidad. Es solamente la elección que hace una cadena comercial de sus blancos. Es decir, afina la llegada del programa porque ha precisado a través de estudios de marketing cuáles son los gustos de diferentes sectores de la población. Así, hay programas muy bien ubicados en la agenda de estas televisoras que van dirigidos al niño, a "la señora de menos de 50 años", porque son precisamente blancos en una estrategia de perpetuación del orden, del statu quo, de comercialización y de consumismo, muy poco critica frente al mundo.

Armand Mattelart: Lo que veo en verdad es que a partir del momento en que los ciudadanos se dan cuenta del lado unilateral de los medios son capaces efectivamente de ya no creer en ellos. Doy un ejemplo sencillo: lo que ocurrió en Francia el 29 de mayo con el referendo para la ratificación del tratado constitucional de la Unión Europea. Es interesante ver que en primer término se pudo establecer que los medios no existen en sí, los medios forman parte de una estructura de poder, son una elite también, que están en colusión con una elite política y económica. Comprobamos que finalmente el $80 \%$ de los periodistas en los medios, desde Le Monde hasta Libération, pasando por Le Figaro, eran medios que estaban en sintonía con el medio político y con el poder económico, en última instancia en sintonía con un dispositivo de poder a favor de la aprobación de la Constitución Europea.

\footnotetext{
${ }^{8}$ Se refiere al Simposio Internacional "Diversidad Cultural, el valor de la diferencia", organizado por el Consejo Nacional de la Cultura, la Coalición Chilena por la Diversidad Cultural y la Universidad Católica, realizado en el Centro de Extensión de esa casa de estudios los días 28 y 29 de junio de 2005.
} 
Entonces, que este dispositivo de poder halla sido desbordado por los ciudadanos, que con ocasión de este referendo trabajaron en nivel de base, pudiendo argumentar, mientras que los que estaban al frente actuaban a partir de temas propagandistas, sin ninguna argumentación, quiere decir que los ciudadanos efectivamente pueden ir mucho más allá, pueden ir por fuera de los cauces que trazan los medios.

Esta toma de conciencia de los ciudadanos franceses indica que hay que discutir. No se puede dejar formar la Unión Europea a partir únicamente de decisiones de tecnócratas. Hay que mirar realmente y discutir cada artículo porque concierne a la vida futura de las nuevas generaciones.

No se puede extrapolar un caso de ese tipo, pero tampoco aceptar como un hecho que no hay que hacer nada en relación a los dispositivos de poder. El problema actual es relegitimar la noción de política publica, porque con el argumento de que finalmente los medios se descrebilizan finalmente vamos a dejar panales enteros de la formación de la opinión publica y de las presentaciones colectivas en barbecho. Y el problema hoy, me parece, incluso si estamos convencidos que los ciudadanos pueden resistir finalmente a estas estructuras concentradas, es que una de las tareas mayores es crear adentro de nuestras instituciones lugares que vigilen, que se vuelvan un actor para que, por ejemplo, un servicio publico se abra a la diversidad.

Ese me parece que es tema central de este siglo, porque el problema no es solamente los medios, es también el uso de las tecnologías. Es verdad que las tecnologías ofrecen efectivamente alternativas, basta recordar los blogs del 11 de marzo en España. La organización de la sociedad civil es fundamental, incluso si el término sociedad civil es muy ambiguo. Es fundamental para repensar la organización mediática, incluso si uno acepta la idea de que finalmente los medios se descrebilizan por sí mismos. Es fundamental para una verdadera política de comunicación.

Aquí en Chile, por ejemplo, el campo del libro es dramático. Cuando uno compara lo que ocurría en los años 70 y 60 y en América Latina, cuando había editoriales como Siglo XXI, o Sudamericana. Todo eso está ahora en manos de grupos españoles, alemanes o franceses, a veces norteamericanos. Yo creo que el libro es una de las partes centrales de la política cultural y de comunicación.

De la misma manera que la globalización ha propulsado una mirada global en la gente, en los líderes globales, es fundamental que este pensamiento global, y a la vez local y nacional, se lo apoderen también los grupos de la sociedad civil, porque si no el riesgo es despertarse con un mundo donde es difícil hablar de democracia.

El problema clave es difícil de discutir, este gran problema clave me parece que es de naturaleza antropológica. Finalmente a qué resistimos y por qué resistimos. Hay que decir que en los años 60 y 70 los psicoanalistas nos habían enseñado que todo régimen, toda fase del capitalismo, exige la construcción de un tipo de personalidad. Sin caer en el "apocalipsismo" hoy en día estamos enfrentados a necesidades que tiene el régimen capitalista para poder proseguir.

Cuando los psicoanalistas te dicen que la mercadotecnia te absorbe, te moviliza el deseo, te moviliza la libido hacia el universo del que hablaba Michelle, el consumismo es realmente una advertencia que hay que tomar en cuenta. Y aquí yo me salgo del problema únicamente de los medios. El problema es saber a qué resistimos: resistimos o no a un programa de televisión o resistimos o no a una sociedad que avanza a través de ejercicios múltiples y a través de su lógica del mercantilismo. 